Volume 8, No.1.6, 2019

International Journal of Advanced Trends in Computer Science and Engineering

Available Online at http://www.warse.org/IJATCSE/static/pdf/file/ijatcse2681.62019.pdf

https://doi.org/10.30534/ijatcse/2019/2681.62019

\title{
Noninvasive Stability Measurement of Linear Voltage Regulator in the Closed-loop Condition
}

\author{
Syukri Zamri, Mohd Hairi Mohd Zaman, Mohd Faisal Ibrahim, Ahmad Asrul Ibrahim, \\ M Marzuki Mustafa \\ Center for Integrated Systems Engineering and Advanced Technologies, Department of Electrical, Electronic and \\ Systems Engineering, Faculty of Engineering and Built Environment, Universiti Kebangsaan Malaysia, 43600 \\ Bangi, Selangor, Malaysia, \\ syukrizamri9@gmail.com, hairizaman@ukm.edu.my, faisal.ibrahim@ukm.edu.my, ahmadasrul@ukm.edu.my, \\ marzuki@ukm.edu.my
}

\begin{abstract}
Few factors may contribute to the stability of a linear voltage regulator (LVR). Among them is the value of the capacitor connected to the output terminal of the LVR, with the primary factor being the parasitic element of the capacitor, namely, equivalent series resistance. The LVR operation is based on the closed-loop system mechanism. LVR stability can be analyzed through two types of responses, namely, transient and frequency. This research focuses on the frequency response of the closed-loop system in terms of the phase margin at the unity gain frequency. Frequency response generates a more accurate outcome than transient response. The transient response can be acquired while the system is in the closed-loop condition, whereas frequency response can only be obtained in the open-loop condition. In this study, a noninvasive method is applied to obtain the LVR frequency response while the system is in the closed-loop condition. LTSpice software is utilized to simulate the LVR circuit. The simulation result is used as a reference for the actual hardware circuitry tests. The obtained outcome shows that the phase margin from the circuit simulation is similar to that of the hardware circuitry. Therefore, the noninvasive stability measurement method is valid for analyzing LVR stability by measuring the phase margin even in the closed-loop condition.
\end{abstract}

Key words: Linear voltage regulator, stability, noninvasive, phase margin.

\section{INTRODUCTION}

Nowadays, voltage regulators are widely used in electronic devices. Therefore, its stability plays a vital role in providing a stable output voltage and minimizing noise as much as possible. The two types of voltage regulators are the linear voltage regulator (LVR) and switching voltage regulator. This research focuses on LVR as it is more straightforward, has less noise, and requires less cost for development than the latter. Electronic devices, which require a constant and stable voltage supply and less noise, use this type of regulator [1], [2].

LVR manufacturers commonly provide LVR characteristics and specifications in their datasheets. One of the most critical parameters is the stable range value of the equivalent series resistance (ESR) of output capacitors [3]-[7]. The output capacitor, connected to the LVR output terminal, has a unique stable range of ESR values for each LVR mode. An optimum ESR range is essential in determining the life expectancy of a capacitor.

LVR stability mainly depends on the ESR, which is the internal parasitic element of every output capacitor connected at the LVR output terminal. ESR values may vary due to aging and temperature factors [1], [2]. Therefore, a stable range of ESR values is required to ensure LVR stability [4], [8]. LVR manufacturers frequently characterize this ESR range in the manufacturing plant. A unique graph, called an ESR tunnel graph in the datasheet, tabulates the outcomes. The graph summarizes the ESR range for a stable LVR.

\subsection{Basic Operation of Linear Voltage Regulator}

Figure 1 depicts a basic LVR circuit. In this example, the LVR receives an input voltage of $5 \mathrm{~V}$ and produces a constant output voltage of $3.3 \mathrm{~V}$. The internal elements of the LVR consist of active components, such as a high gain differential amplifier that controls the pass element, either MOSFET or BJT transistors. The differential amplifier compares the output voltage through feedback resistors using a reference voltage [9], [10].

LVRs have been widely used in electronic devices where the load requires a constant and stable input voltage. One of the factors that affect LVR stability is the output capacitor with specific ESR values, which is connected to the LVR output terminal. The LVR datasheet describes stable ESR values to reach stability. The output voltage produced by the LVR must be constant and stable even if a disturbance occurs. 


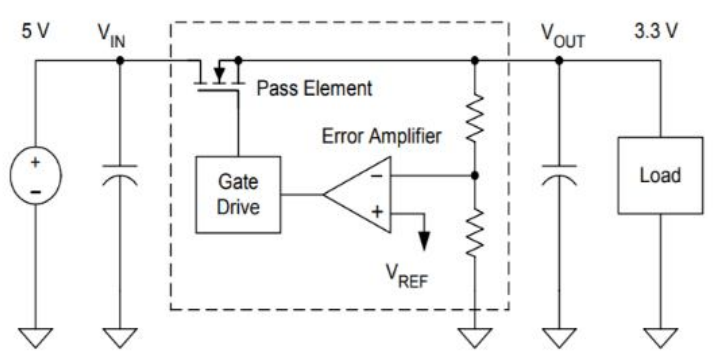

Figure 1: Basic Circuit of an LVR

\subsection{ESR Characterization}

Output capacitors connected to the output terminal of LVRs are mostly the electrolytic type due to its popularity and low cost. Such capacitors must contain a stable range of ESR for the LVR to produce a stable output voltage. Figure 2 presents an example of the ESR tunnel graph, which is a graph of the output capacitor ESR versus the output current of an LVR. The LVR manufacturers perform this ESR characterization during LVR production for user reference. Given the extensive range of resistors connected in series to the output capacitor for simulating the ESR values, this characterization is manually conducted, an approach which consumes considerable time. When the characterization is accomplished, the result is specified as an ESR tunnel graph in the datasheet [7], [11]-[13].

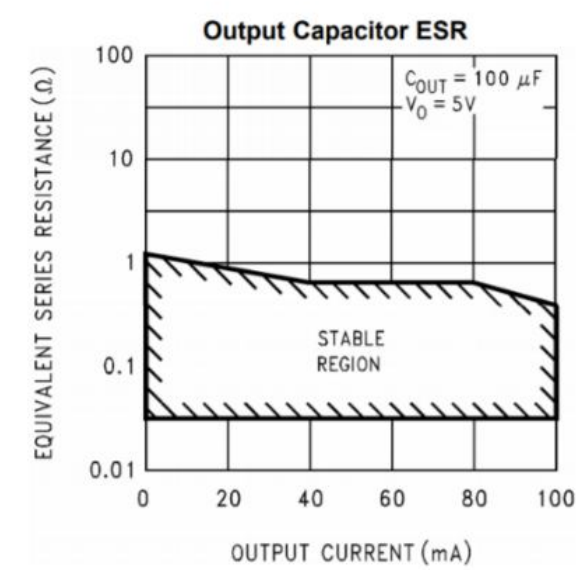

Figure 2: Example of an ESR Tunnel Graph for an LVR with an Output Capacitor of $100 \mu \mathrm{F}$

The ESR is characterized based on LVR stability. The output capacitor with a specific ESR is the main parameter of LVR stability. Only a specific value of the ESR can stabilize the LVR and produces a stable output voltage. Moreover, the high demand for LVR is due to its application in most current electronic devices; therefore, its stability criterion is crucial [14]-[16].

\subsection{Stability Analysis of LVR}

LVR stability can be analyzed through two types of responses: (a) the load transient response in the time domain or (b) the frequency response in the frequency domain. The load transient response of the LVR can be ascertained while the system remains in a closed-loop condition. Meanwhile, the frequency response of the LVR can be obtained when the system is in the open-loop condition. However, stability measurement based on the frequency response via a phase margin generates an accurate stability indicator of the system.

As shown in Figure 1, the LVR system is typically packaged in a closed-loop condition; therefore, yielding its load transient response is straightforward [17]. Frequency response can yield more accurate stability measurement than transient response because it indicates the phase margin of the system [18]. However, the frequency response can only be obtained while the system is in the open-loop condition, during which breaking the loop in the actual LVR is challenging.

Prior research has broken the loop of the LVR system through the invasive method. However, this method is meticulous and time-consuming. Hence, a noninvasive method is used in the present research to obtain the LVR frequency response while the system remains in the closed-loop condition [5], [19].

\section{METHODS}

Figure 3 shows the overall flowchart for the noninvasive method conducted in this research. The LTSpice circuit simulation software was used to simulate the LVR circuit and identify its frequency response through invasive and noninvasive techniques. This work analyzed two test circuits. One is the LVR circuit that was constructed using discrete components. Another test circuit was based on commercial LVR.

\subsection{LVR Circuit Constructed Using Discrete Components}

First, the LVR circuit was constructed using several discrete components to compare invasive and noninvasive methods. The invasive method was implemented by connecting a coupled inductor and capacitor to the output of the differential amplifier. Figure 4 depicts the LVR circuit for the invasive technique to obtain the frequency response. The coupled inductor and capacitor were utilized to close the loop in the DC mode and open the circuit in the AC mode. The simulation shorts all inductors and opens all capacitors to recognize the DC bias point connected to the power amplifier. Therefore, when the simulation recognizes the DC bias point, the system will be in the closed-loop condition through the coupled inductor. However, the system is in the open-loop through the coupled capacitor, thereby causing the circuit to be in the open-loop condition and eventually obtaining the frequency response. 


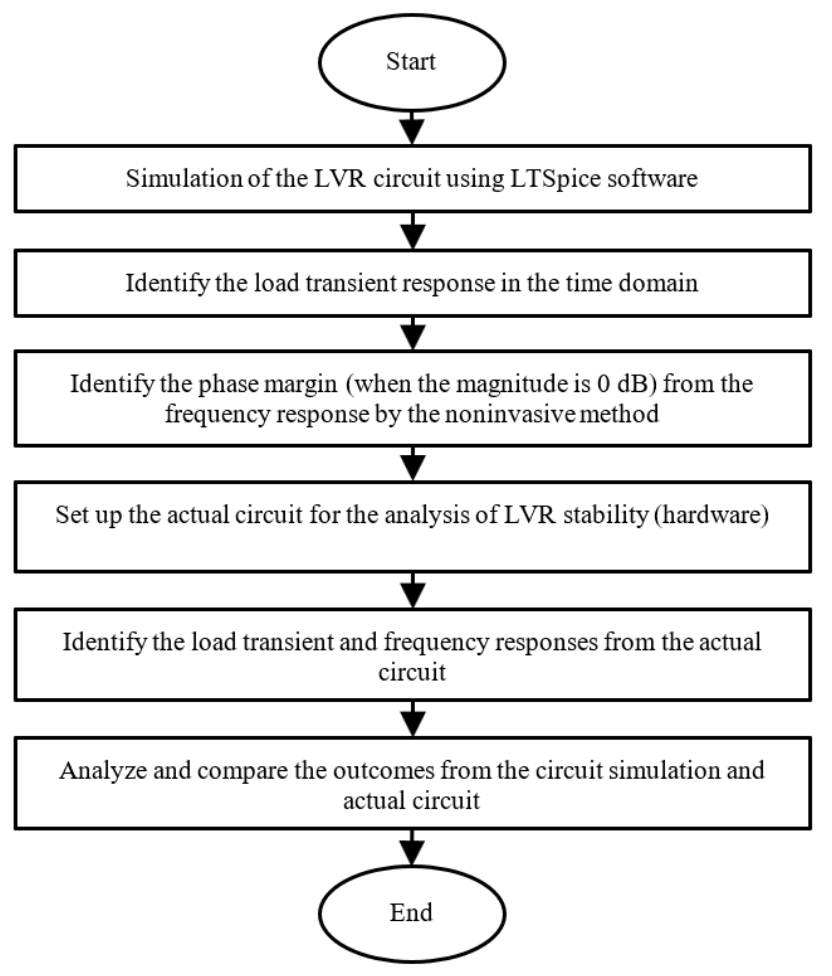

Figure 3: Flowchart of Noninvasive Method

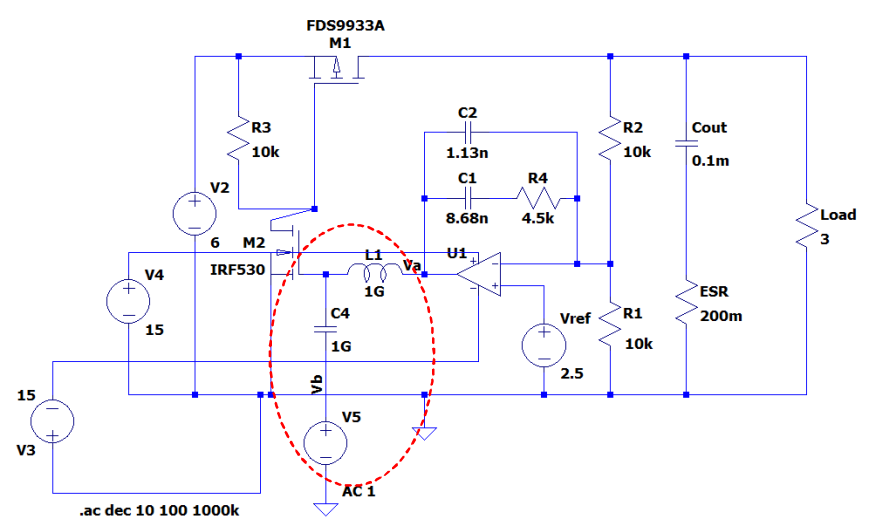

Figure 4: Invasive Method to Obtain the Frequency Response of the LVR Circuit Constructed Using Discrete Components

After obtaining the frequency response of the LVR by using the invasive technique, the noninvasive technique for the same LVR circuit was implemented. The noninvasive technique refers to a technique to obtain the frequency response of the system (in this case, the LVR system) without breaking its loop. Figure 5 shows the noninvasive technique for obtaining the frequency response of a simple LVR circuit constructed using discrete components and is similar to the circuit depicted in Figure 4.

A small signal was injected into the LVR system in the noninvasive method to measure the signal that enters and exits the loop [20]-[24]. The system loop is broken at a low impedance output point. The point is usually above the upper resistor, which is located in the feedback path of the LVR circuit. Therefore, one side of the injection point should have high impedance, and other sides should have low impedance. Such a condition creates a satisfactory injection point because a transfer function of the system can be obtained. These points are labeled points $\mathrm{v}_{\mathrm{a}}$ and $\mathrm{v}_{\mathrm{b}}$. A small resistor with a value of 5 $\Omega$ was used to separate these two points electrically. The reason is that a small resistor will negligibly influence the output voltage of the LVR, thereby allowing the establishment of system measurement.

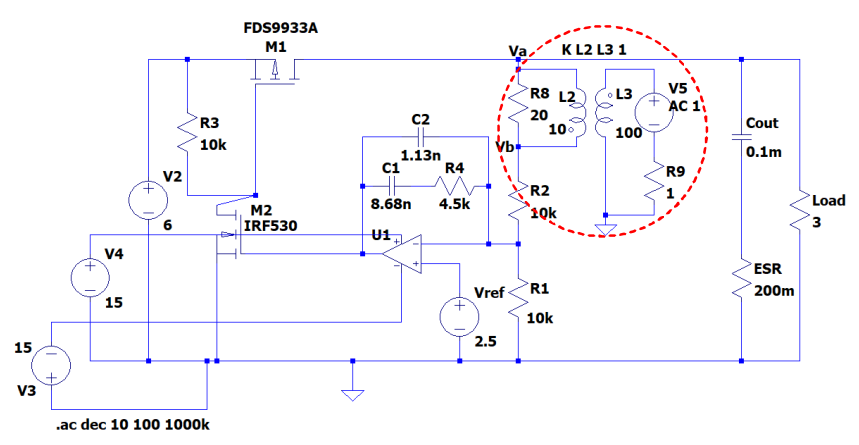

Figure 5: Noninvasive Method to Obtain the Frequency Response of the LVR Circuit Constructed Using Discrete Components

When everything has been set up, as shown in Figure 5, the circuit is powered up, and the signal is passed through point $\mathrm{v}_{\mathrm{b}}$ and out through Point $\mathrm{v}_{\mathrm{a}}$. The function generator connected at the secondary side of the injection transformer, L3, was set to produce a sine wave signal for an easy phase margin measurement. If test points $\mathrm{v}_{\mathrm{a}}$ and $\mathrm{v}_{\mathrm{b}}$ were connected to the oscilloscope channels, then a sinusoidal wave will appear on both channels. However, both channels will not show the same sinusoidal wave. That is, both channels will have amplitude gains different from each other. The frequency on the function generator was adjusted until the same amplitude was on both channels. At this point, the gain for the system's loop is 1 or the unity gain. The adjusted frequency when the amplitudes are equivalent is the frequency of the $0 \mathrm{~dB}$ crossover of the system or the unity gain frequency.

Both sine waves from the two channels have phase differences from each other, where one of the sine waves is shifted to another. This amount of phase shift represents the value of the phase margin of the system. If invasive and noninvasive approaches produce the same results, then the noninvasive method is proven to be valid in this research.

\subsection{LVR Circuit Constructed Using Commercial LVR}

The second test circuit was tested, wherein the LVR circuit was constructed using a commercial LVR of model LT1963A from Linear Technology [25]. This circuit was simulated in the LTSpice software and subsequently constructed in the hardware circuitry form. Data were collected from these two approaches. Figure 5 depicts this LT1963A LVR circuit for testing and validating the noninvasive stability measurement method in this work. The manufacturer datasheet indicates 
that the range of the input voltage $\left(\mathrm{V}_{\text {in }}\right)$ should be from $2.2 \mathrm{~V}$ to $20 \mathrm{~V}$. The output voltage $\left(\mathrm{V}_{\text {out }}\right)$ of LT1963A can be varied by using equation as follows,

$$
V_{O U T}=V_{A D J}\left(1+R_{2} / R_{1}\right)+I_{A D J} R_{2}
$$

where $V_{A D J}=1.21 \mathrm{~V}$ and $I_{A D J}=3 \mu \mathrm{A}$. The output voltage can be determined by selecting the appropriate values of $\mathrm{R}_{1}$ and $\mathrm{R}_{2}$. The selected output voltage should be in the range of 1.21 to $20 \mathrm{~V}$. The selected output capacitor in this research was 10 $\mu \mathrm{F}$, and based on the datasheet, the maximum value of the ESR for a stable LVR was $3 \Omega$.

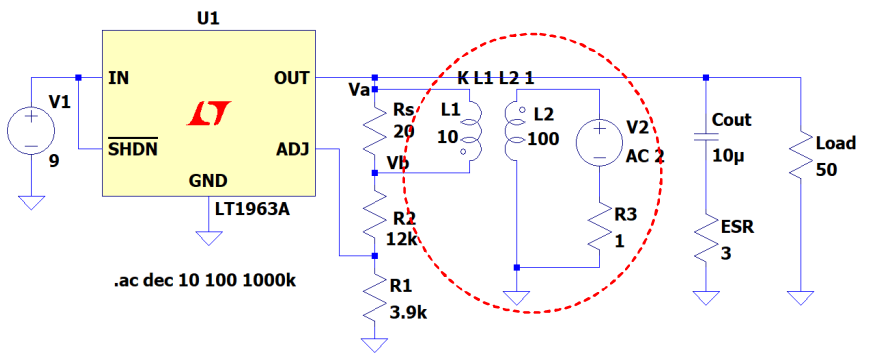

Figure 6: Noninvasive Method to Obtain the Frequency Response of the LVR Circuit Constructed Commercial LVR

The ESR values varied from $2 \Omega$ to $4 \Omega$ per load with an increment of $0.1 \Omega$. The loads used were $3.33 \Omega, 10 \Omega$, and 5 $\mathrm{k} \Omega$. The output current calculated based on Ohm's law was $1.5 \mathrm{~A}, 0.5 \mathrm{~A}$, and $1 \mathrm{~mA}$. For the hardware circuitry, the same methods were applied by using the same components utilized in the LTSpice simulation. A simple resistor with values ranging from $2 \Omega$ to $4 \Omega$ was connected in series with the output capacitor to represent the ESR value. When the LVR characteristics were obtained from the simulation and hardware circuitry approaches, the results were compared for validation.

\section{RESULTS AND DISCUSSION}

In this research, the output voltage of an LVR is considered stable if it has a maximum of three oscillations in its transient response. Furthermore, the output voltage must have a maximum swing of $\pm 0.2 \mathrm{~V}$. Conversely, in the frequency response, the output voltage should have a minimum phase margin of $45^{\circ}$. A phase margin value of more than $45^{\circ}$ indicates a further stable LVR. Figure 7 and 8 show the frequency responses obtained from invasive and noninvasive methods, respectively, for the LVR circuit constructed using discrete components. Both approaches were implemented and compared to validate the noninvasive method proposed in this work, even though it did not represent an actual LVR. Figure 7 and 8 show that the invasive and noninvasive methods yield a phase margin of $25.35^{\circ}$. These outcomes prove that the noninvasive method is valid and can be used to obtain the frequency response of the LVR system, even one in a closed-loop condition.

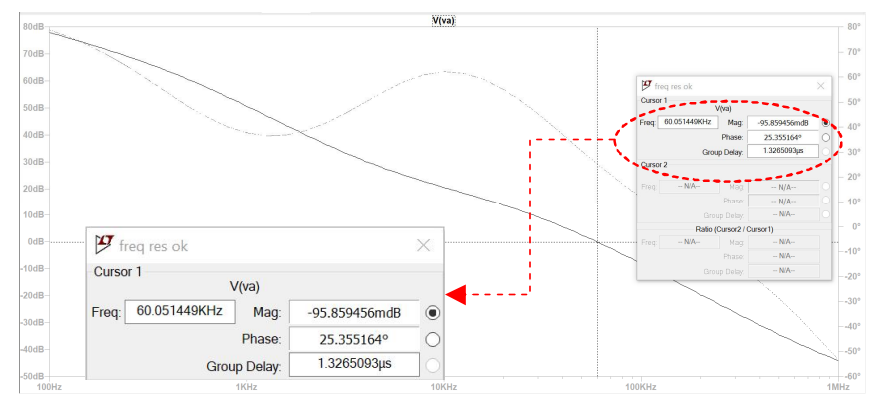

Figure 7: Frequency Response Obtained from the Invasive Method for an LVR Circuit Constructed Using Discrete Components

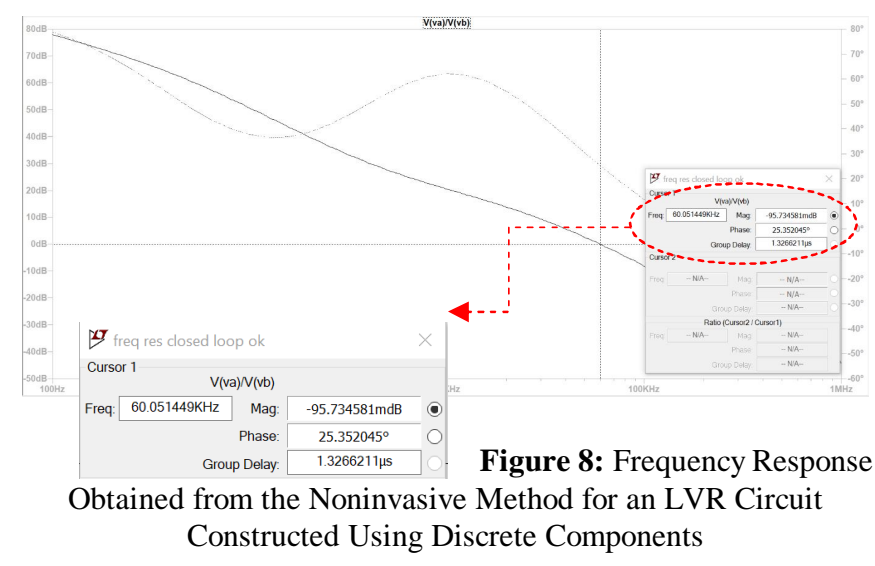

The next approach was conducted using the LVR model LT1963A. Figure 9 and 10 show the load transient responses obtained from LT1963A through software simulation and hardware circuitry, respectively. The load transient response through software simulation and hardware circuitry yields the undershoot of $0.216 \mathrm{~V}$ and $0.182 \mathrm{~V}$, respectively. These outcomes show that the LVR is stable, but this time-domain approach is not accurate compared with the frequency response approach. The frequency response approach measures the system's phase margin, as shown in Figure 11 and 12. Correctly, Figures 11 and 12 present the frequency response yield from software simulation and hardware circuitry, respectively, for the LT1963A LVR.

Figure 11 presents the difference between the peak-to-peak values from both waveforms in the time domain $(\mu \mathrm{s})$. It is divided by a full cycle's period, which is $10.95 \mu$ s, and multiplied by $360^{\circ}$ to obtain the value in degrees for indicating the phase margin. Figure 13 shows the period for one full cycle. For example, the computed phase margin is $(2.716 \mu / 10.95 \mu \mathrm{s}) * 360^{\circ}=89.23^{\circ}$. The phase margins for software simulation and hardware circuitry are $89.23^{\circ}$ and $101.6^{\circ}$, respectively. The accuracy of the noninvasive stability measurement based on the frequency response is $87.8 \%$. This deviation may be due to the inaccurate measurement of ESR values in the output capacitor and the actual wiring connection. However, this outcome is significant for measuring LVR stability through the phase margin. All phase margin comparison is depicted in Table 1. 


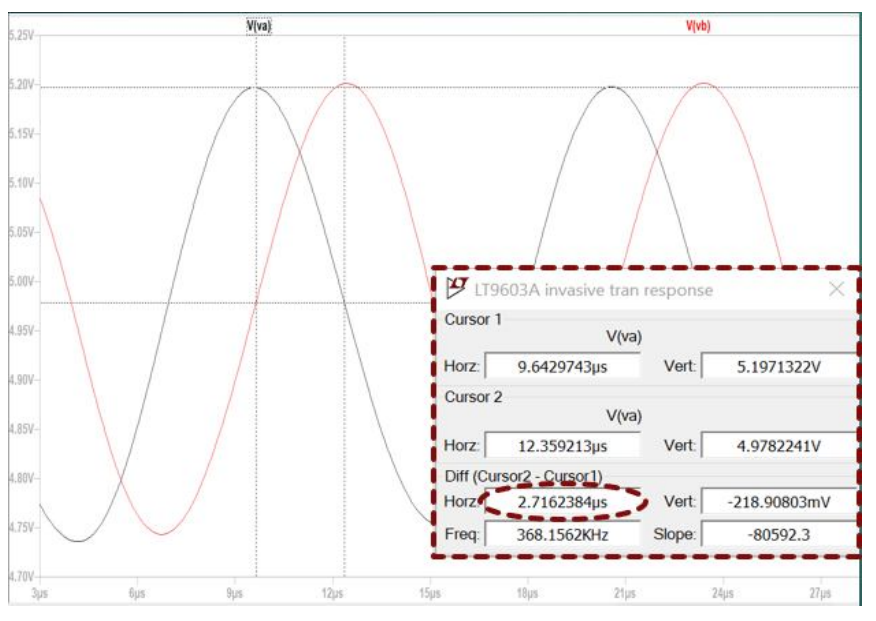

Figure 11: Frequency Response from the Software Simulation of an LT1963A Circuit

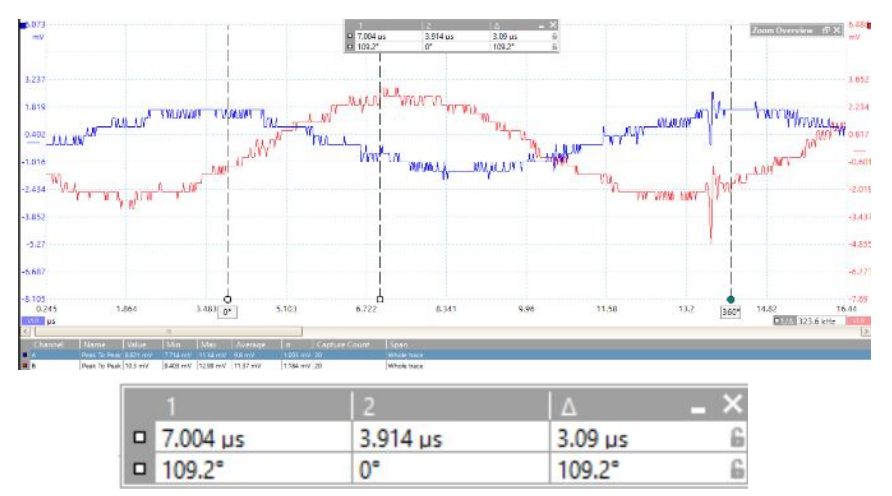

Figure 12: Frequency Response from the Hardware Circuitry of an LT1963A Circuit

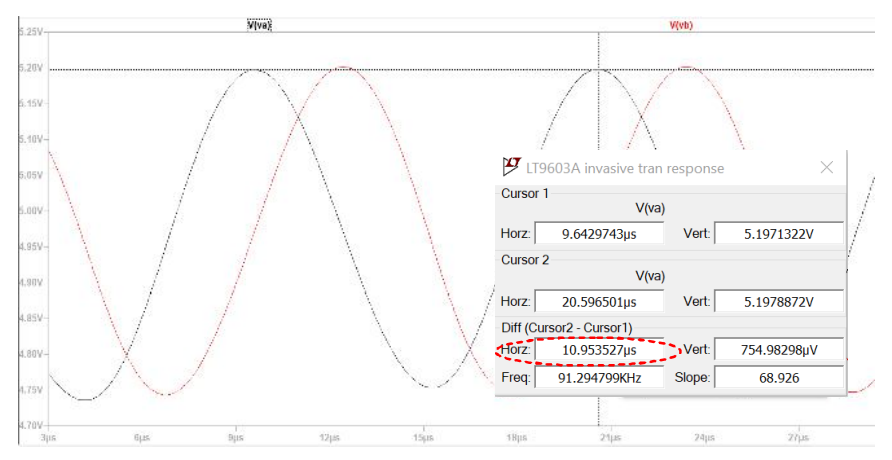

Figure 13: Period for One Full Cycle of the Output Voltage from an LT1963A Circuit

Table 1: Phase Margin Comparison

\begin{tabular}{|c|c|c|c|}
\hline \multirow{2}{*}{ LVR circuit type } & \multicolumn{2}{c|}{ Phase margin } \\
\cline { 2 - 4 } & Invasive & Noninvasive \\
\hline \multicolumn{2}{|c|}{ Discrete component } & $25.35^{\circ}$ & $25.35^{\circ}$ \\
\hline \multirow{2}{*}{$\begin{array}{c}\text { LT1963A } \\
\text { (commercial) }\end{array}$} & Simulation & - & $89.23^{\circ}$ \\
\cline { 2 - 4 } & Hardware & - & $101.60^{\circ}$ \\
\hline
\end{tabular}

\section{CONCLUSION}

The noninvasive method for measuring LVR stability is proven to be valid and can be used to identify the phase margin from the frequency response of the LVR, even one in the closed-loop condition. The ESR and output capacitor considerably contribute to LVR stability; thus, ESR measurement in the output capacitor is crucial before analyzing LVR stability.

\section{ACKNOWLEDGEMENT}

This work was financially supported by Ministry of Education Malaysia under the Fundamental Research Grant Scheme no. FRGS/1/2019/TK04/UKM/03/1.

\section{REFERENCES}

1. G. A. Rincon-Mora. Analog IC Design with Low-Dropout Regulators, United States of America: McGraw-Hill, 2014, ch. 1, pp. 2-6.

2. K. H. Chen. Power Management Techniques for Integrated Circuit Design, Singapore: John Wiley \& Sons, 2015, ch. 2, pp. 28-29.

3. B. C. Bao, X. Zhang, and J. P. Xu. Critical ESR of output capacitor for stability of fixed off-time controlled buck converter, Electronic Letters, vol. 49, no. 4, pp. 287-288, 2013. https://doi.org/10.1049/el.2012.2607

4. B. S. Lee. Understanding the stable range of equivalent series resistance of an LDO regulator, Analog Applications Jour., vol. 1, pp. 14-17, 1999.

5. S. Sandler. Simple method to determine ESR requirements for stable regulators, Picotest Application Note, pp. 1-6, 2011.

6. K. Subasinghage, K. Gunawardane, N. Kularatna, and T. $\mathrm{T}$. Lie. Selection of the stable range of the equivalent series resistance (ESR) of the output capacitor for a SCALDO regulator, in Proc. IEEE 27th Int. Symposium on Industrial Electronics, 2018, pp. 1359-1364.

7. A. H. Musa, M. H. M. Zaman, R. Mohamed, and M. M. Mustafa. Characterization of voltage regulators by automated equivalent series resistance, in Proc. 2014 IEEE Conf. on Systems, Process and Control, 2014, pp. 68-72.

8. J. Falin. ESR, stability and the LDO regulator, Texas Instruments Application Report, pp. 1-6, 2002.

9. C. K. Chava, and J. Silva-Martinez. A frequency compensation scheme for LDO voltage regulators, IEEE Trans. on Circuits and Systems I: Regular Papers, vol. 51, no. 6, pp. 1041-1050, 2004.

https://doi.org/10.1109/TCSI.2004.829239

10. A. Garimella, P. R. Surkanti, and P. M. Furth. Pole-zero analysis of low-dropout (LDO) regulatos: A tutorial overview, in Proc. IEEE Int. Conf. on VLSI Design, 2012, pp. 131-136.

11. M. H. M. Zaman, M. M. Mustafa, and A. Hussain. Critical equivalent series resistance estimation for 
voltage regulator stability using hybrid system identification and neural network, Int. Jour. on Advanced Sci., Eng. and Information Technology, vol. 7, no. 4, pp. 1381-1388, 2017.

12. M. H. M. Zaman, M. M. Mustafa, M. A. Hannan, and A. Hussain. Neural network based prediction of stable equivalent series resistance in voltage regulator characterization, Bull. of Electrical Eng. and Informatics, vol. 7, no. 1, pp. 134-142, 2018.

13. M. H. M. Zaman, M. M. Mustafa, and A. Hussain. Estimation of voltage regulator stable region using radial basis function neural network, Jour. of Telecommunication, Electronic and Computer Eng., vol. 10, no. 2-8, pp. 63-66, 2018.

14. A. Ciprut, and E. G. Friedman. On the stability of distributed on-chip low dropout regulators, in Proc. 2017 IEEE 60th Int. Midwest Symposium on Circuits and Systems, 2017, pp. 217-220.

15. T. Coulot, E. Lauga-Larroze, J. M. Fournier, M. Alamir, and F. Hasbani. Stability analysis and design procedure of multiloop linear LDO regulators via state matrix decomposition, IEEE Trans. on Power Electronics, vol. 28, no. 11, pp. 5352-5363, 2013.

16. S. Lai, B. Yan, and P. Li. Localized stability checking and design of IC power delivery with distributed voltage regulators, IEEE Trans. on Computer-Aided Design of Integrated Circuits and Systems, vol. 32, no. 9, pp. 1321-1334, 2013.

https://doi.org/10.1109/TCAD.2013.2256393

17. A. D. M. Africa, P. B. T. Arevalo, A. S. Publico, and M. A. A. Tan. Time response analysis of control systems, Int. Jour. of Advanced Trends in Computer Science and Eng., vol. 8, no. 4, pp. 1416-1420, 2019.

https://doi.org/10.30534/ijatcse/2019/59842019

18. A. D. M. Africa, P. B. T. Arevalo, A. S. Publico, and M. A. A. Tan. Modeling in the frequency domain of control systems, Int. Jour. of Advanced Trends in Computer Science and Eng., vol. 8, no. 4, pp. 1427-1432, 2019. https://doi.org/10.30534/ijatcse/2019/61842019

19. Rohm Semiconductor. Simple test method for estimating the stability of linear regulators, Rohm Semiconductor Application Note, pp. 1-4, 2016.

20. C. Basso. Designing Control Loops for Linear and Switching Power Supplies: A Tutorial Guide, United States of America: Artech House, 2012, ch. 9, pp. 519-527.

21. R. D. Middlebrook. Measurement of loop gain in feedback systems, Int. Jour. of Electronics, vol. 38, no. 4, pp. 485-512, 1975.

22. R. W. Erickson, and D. Maksimovic. Fundamentals of Power Electronics, United States of America: Kluwer, 2001, ch. 9, pp. 362-368.

23. V. Vorperian. Fast Analytical Techniques for Electrical and Electronic Circuits, Cambridge: Cambridge University Press, 2002, ch. 5, pp. 175-179.

https://doi.org/10.1017/CBO9780511613791
24. Y. Panov, and M. Jovanovic. Small-signal measurements in switching power supplies, in Proc. Applied Power Electronics Conf., 2004, pp. 770-776.

25. M. H. M. Zaman, M. M. Mustafa, and A. Hussain. Black-box modeling of low dropout voltage regulator based on two-port network parameter identification, in Proc. 2015 IEEE 11th Int. Colloquium on Signal Processing and Its Applications, 2015, pp. 70-75. 